\title{
Dynamic Analysis for a Fractional-Order Autonomous Chaotic System
}

\author{
Jiangang Zhang, ${ }^{1}$ Juan Nan, ${ }^{1}$ Wenju Du, ${ }^{2}$ Yandong Chu, ${ }^{1}$ and Hongwei Luo \\ ${ }^{1}$ School of Mathematics and Physics, Lanzhou Jiaotong University, Lanzhou 730070, China \\ ${ }^{2}$ School of Traffic and Transportation, Lanzhou Jiaotong University, Lanzhou 730070, China \\ Correspondence should be addressed to Jiangang Zhang; zhangjg7715776@126.com
}

Received 23 January 2016; Accepted 26 June 2016

Academic Editor: Viktor Avrutin

Copyright (c) 2016 Jiangang Zhang et al. This is an open access article distributed under the Creative Commons Attribution License, which permits unrestricted use, distribution, and reproduction in any medium, provided the original work is properly cited.

\begin{abstract}
We introduce a discretization process to discretize a modified fractional-order optically injected semiconductor lasers model and investigate its dynamical behaviors. More precisely, a sufficient condition for the existence and uniqueness of the solution is obtained, and the necessary and sufficient conditions of stability of the discrete system are investigated. The results show that the system's fractional parameter has an effect on the stability of the discrete system, and the system has rich dynamic characteristics such as Hopf bifurcation, attractor crisis, and chaotic attractors.
\end{abstract}

\section{Introduction}

The idea of fractional-order calculus (FOC) has been well known since the development of the regular calculus. The significant progress on FOC has been witnessed because the FOC has a wide range of applications in diffusion. In the past decades, chaotic systems have become a focal point of renewed interest for many researchers. And we can find these nonlinear systems in various natural and man-made systems, which are known to have great sensitivity to initial conditions. And we can find chaos phenomenon in various natural and man-made systems, and these chaotic systems have great sensitivity to initial conditions. Because differential equations with fractional order can be applied in many areas of science and engineering, they attracted many researchers' attention and their complex behaviors have been widely studied in recent years. Nowadays, there is increasing interest in the subject of a fractional model which can give a more realistic interpretation of practical phenomena. Furthermore, many systems in interdisciplinary fields can be described by the fractional differential equations, such as turbulence, electromagnetism, signal processing, and quantum evolution of complex systems. It is also demonstrated that some fractional-order differential systems behave chaotically or hyperchaotically, such as fractional-order Chen system [1-3] and fractional-order Lorenz system $[4,5]$. The fractionalorder equations are more suitable than integer-order ones to describe the biological, economic, and social systems where memory effects are important [6]. More recently, it has been found that some differential systems with fractional order possess chaotic behavior [7-16]. And many Lorenzlike or Lorenz-based chaotic systems were proposed and investigated. Some classical 3D autonomous chaotic systems have three particular fixed points: one saddle and two unstable saddle foci [17]. And the other 3D chaotic systems have two unstable saddle foci $[18,19]$. Yang and Chen [20] found another 3D chaotic system with three fixed points: one saddle and two stable equilibriums. However, an increasing number of three-dimensional chaotic systems have been found these years in many physical and engineering fields. Nonlinear dynamics of a semiconductor laser subject to optical injection is currently a hot research field due to its rich physics and complexity as well as its potential applications in communications [21, 22]. Momani et al. [23] applied the multistep generalized differential transform method to solve the fractional-order multiple chaotic FitzHugh-Nagumo (FHN) neurons model. They illustrated the algorithm by studying the dynamics of three coupled chaotic FHN neurons equations with different gap junctions under external electrical stimulation, and the fractional derivatives are described in 
the Caputo sense. The numerical simulation results show that only few terms are required to deduce the approximate solutions which are found to be accurate and efficient.

Optically injected semiconductor lasers revealed amazingly rich behaviors like stable locking, coexistence of attractors, quasiperiodicity, instabilities, pulsations, and many routes to chaos such as period-doubling cascades, intermittency, breakup of tori, and homoclinic and heteroclinic tangencies [24-30], which deserve further systematic investigation. Wieczorek et al. [31] studied the dynamics and bifurcations of a single-mode semiconductor laser with optical injection and investigated the dependence of the dynamics on the injected field strength $K$ and its detuning $\omega$ from the unperturbed laser resonant frequency and the linewidth enhancement factor $\alpha$ which seem to have the most significant influence on the dynamics. Chlouverakis and Adams [32] discussed the dynamics of a semiconductor laser subject to optical injection using the method of the Largest Lyapunov Exponent (LLE) and used this model as one of three examples to investigate the relation between the correlation $\left(D_{2}\right)$ and the Kaplan-Yorke dimension $\left(D_{\mathrm{KY}}\right)$ of three-dimensional chaotic flows. Chu et al. [33] studied the dynamical properties of the semiconductor laser subject to optical injection and a new almost-Hamilton chaotic system with very high Lyapunov dimensions is constructed and investigated. The aim of this paper is to investigate the dynamical behavior of a discretization fractional order of a modified optically injected semiconductor lasers model.

The paper is organized as follows. In Section 2, we give some related preliminaries. We present the fractional-order semiconductor lasers model and introduce a discretization process to discretize a modified fractional-order optically injected semiconductor lasers model in Section 3. In Section 4 , a sufficient condition for existence and uniqueness of the solution of the fractional-order system is investigated and the equilibrium points and their asymptotic stability in the fractional-order model and its discrete counterpart are also studied. In Section 5, we give some numerical simulations, which not only illustrate our results with the theoretical analysis, but also exhibit the complex dynamical behaviors of the discretization fractional order of a modified optically injected semiconductor lasers model. Finally, conclusions are given in Section 6.

\section{Preliminaries}

The Caputo definition of fractional derivative [34] is given as follows:

$$
D^{\alpha} f(t)=I^{l-\alpha} f^{(l)}(t), \quad \alpha>0,
$$

where $l$ is the least integer which is not less than $a$ and $I^{\theta}$ is the Riemann-Liouville integral operator of order $h$ which is given by

$$
I^{\theta} u(t)=\frac{1}{\Gamma(\theta)} \int_{0}^{t}(t-\tau)^{(\theta-1)} u(\tau) d \tau, \quad \theta>0,
$$

where $\Gamma(\theta)$ is Euler's Gamma function. The operator $D^{\alpha}$ is termed "Caputo differential operator of order $\alpha$." In $[35,36]$, the geometric and physical interpretations of the fractional derivatives were investigated.

The stability conditions and their applications to fractional-order differential equations were reported in [37-40]. The local stability of the equilibrium points of a linearized fractional-order system can be obtained from the following Matignon result [37]:

$$
\left|\arg \left(\lambda_{i}\right)\right|>\frac{\alpha \pi}{2}, \quad(i=1,2,3)
$$

where $\lambda_{1}, \lambda_{2}$, and $\lambda_{3}$ are the eigenvalues of the system. Then, we consider the following nonlinear autonomous fractionalorder system:

$$
D^{\alpha} X(t)=f(X(t)), \quad X(0)=X_{0},
$$

where $X(t)=\left(x_{1}, x_{2}, x_{3}\right)^{T} \in R^{3}$ and $f: R^{3} \rightarrow R^{3}$ is a nonlinear vector function in terms of $X$. The Jacobian matrix evaluated at the equilibrium point $X^{*}=\left(x_{1}^{*}, x_{2}^{*}, x_{3}^{*}\right)$ is

$$
J\left(X^{*}\right)=\left.\left(\frac{\partial f_{i}}{\partial x_{j}}\right)_{i j}\right|_{X=X^{*}} .
$$

Hence, we have the following lemma [41].

Lemma 1. If all the eigenvalues $\lambda_{1}, \lambda_{2}$, and $\lambda_{3}$ of the equilibrium point $X^{*}$ of system (4) satisfy Matignon's condition (3), then $X^{*}$ is locally asymptotically stable.

\section{Fractional-Order Semiconductor Lasers Model and Its Discretization}

Chlouverakis and Adams [32] present a modified optically injected semiconductor lasers model as follows:

$$
\begin{aligned}
\frac{\partial E_{x}}{\partial t} & =K+\frac{1}{2} E_{x}+\left(\omega-\frac{1}{2} \alpha n\right) E_{y}, \\
\frac{\partial E_{y}}{\partial t} & =-\left(\omega-\frac{1}{2} \alpha n\right) E_{x}+\frac{1}{2} E_{y} n, \\
\frac{\partial n}{\partial t} & =-2 \Gamma n-(1+2 B n)\left(E_{x}^{2}+E_{y}^{2}-1\right),
\end{aligned}
$$

where the complex electric field $E=E_{x}+i E_{y}$ and $n$ is the normalized population inversion, $K$ is the injection strength, $\omega$ is the detuning, and $\alpha$ is the linewidth enhancement factor. And the parameters $B=\omega_{R} /\left(2 \Gamma_{0}\right)$ and $\Gamma=\Gamma_{N} /\left(2 \omega_{R}\right)+B, \Gamma_{0}$ is the inverse photon lifetime, $\Gamma_{N}$ is the inverse electron lifetime, and $\omega_{R}$ is the angular relaxation oscillation frequency. For simplification, in this paper, we established a dimensionless modified optically injected semiconductor lasers model by dimensionless method:

$$
\begin{aligned}
& \dot{x}=a+z(x-b y), \\
& \dot{y}=z(b x-c y), \\
& \dot{z}=1-x^{2}-y^{2},
\end{aligned}
$$




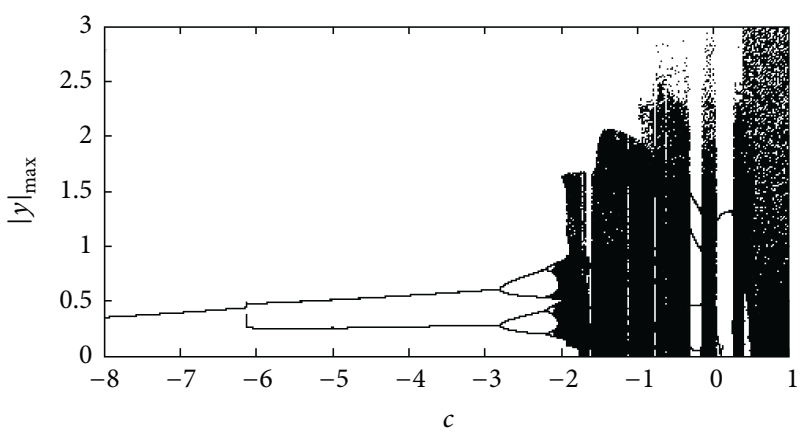

(a)

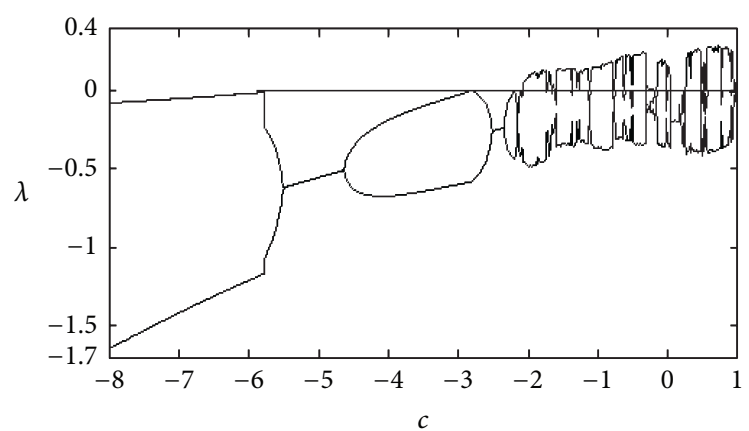

(b)

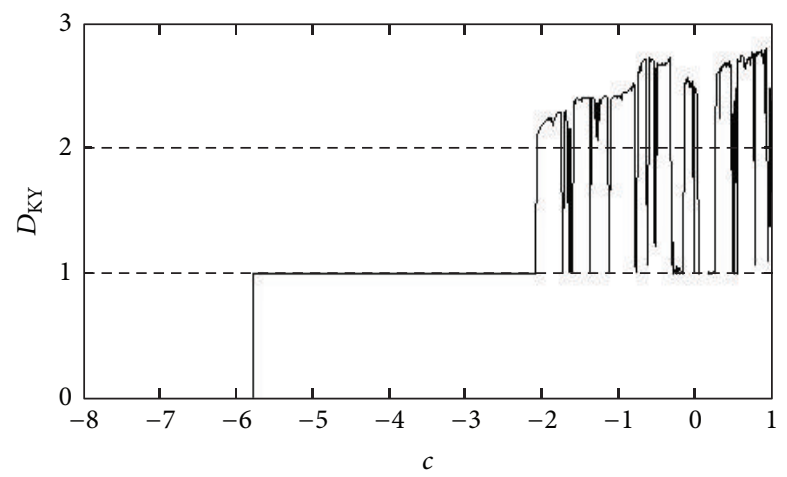

(c)

FIGURE 1: (a) Bifurcation diagram for specific values set $(a=0.4, b=3)$ versus $c$, (b) Lyapunov-exponent spectrum against $c$ corresponding to the bifurcation diagram of (a), and (c) Kaplan-Yorke dimension versus the control parameter $c$ corresponding to the bifurcation diagram of (a).

where $(x, y, z) \in R^{3}$ are the state variables and $(a, b, c) \in R^{3}$ are real parameters. The bifurcation diagram of $|y|_{\max }$ versus control parameter $c$, the Lyapunov-exponent spectrum, and the Kaplan-Yorke dimension for specific values set $(a=$ $0.4, b=3$ ) versus the bifurcation parameter $c$ on the open interval $(-8,1)$ are shown in Figures 1(a)-1(c), respectively.

System (7) is dissipative and exhibits a chaotic attractor when the parameter values $a=0.4, b=3$, and $c=0.73$. Figure 2 shows the chaotic attractor and the Poincare map in $x-z\{y=0\}$ plane of chaotic system (7).

Next, we will investigate the following fractional order of a modified optically injected semiconductor lasers model:

$$
\begin{aligned}
& \frac{d^{\alpha} x}{d t^{\alpha}}=a+z(x-b y), \\
& \frac{d^{\alpha} y}{d t^{\alpha}}=z(b x-c y), \\
& \frac{d^{\alpha} z}{d t^{\alpha}}=1-x^{2}-y^{2},
\end{aligned}
$$

where $\alpha$ is the fractional order satisfying $0<\alpha \leq 1$ and $d^{\alpha} / d t^{\alpha}$ is in the sense of the Caputo fractional derivative.

And the discretization process of the fractional-order modified optically injected semiconductor lasers model is given as follows.
Assume that $x(0)=x_{0}, y(0)=y_{0}$, and $z(0)=z_{0}$ are the initial conditions of system (8). So, the discretization of system (8) with piecewise constant arguments is given as

$$
\begin{aligned}
& D^{\alpha} x(t)=a+z\left(\left[\begin{array}{l}
t \\
s
\end{array}\right] s\right)\left(x\left(\left[\begin{array}{l}
t \\
s
\end{array}\right] s\right)-b y\left(\left[\begin{array}{l}
t \\
s
\end{array}\right] s\right)\right), \\
& D^{\alpha} y(t)=z\left(\left[\begin{array}{l}
t \\
s
\end{array}\right] s\right)\left(b x\left(\left[\frac{t}{s}\right] s\right)-c y\left(\left[\frac{t}{s}\right] s\right)\right), \\
& D^{\alpha} z(t)=1-\left(x\left(\left[\begin{array}{l}
t \\
s
\end{array}\right] s\right)\right)^{2}-\left(y\left(\left[\begin{array}{l}
t \\
s
\end{array}\right] s\right)\right)^{2} .
\end{aligned}
$$

First, we let $t \in[0, s)$, so $t / s \in[0,1)$. Thus, we obtain

$$
\begin{aligned}
& D^{\alpha} x(t)=a+z_{0}\left(x_{0}-b y_{0}\right), \\
& D^{\alpha} y(t)=z_{0}\left(b x_{0}-c y_{0}\right), \\
& D^{\alpha} z(t)=1-x_{0}^{2}-y_{0}^{2},
\end{aligned}
$$

and the solution of (10) is reduced to

$$
\begin{aligned}
x_{1}(t) & =x_{0}+I^{\alpha}\left(a+z_{0}\left(x_{0}-b y_{0}\right)\right) \\
& =x_{0}+\frac{t^{\alpha}}{\alpha \Gamma(\alpha)}\left(a+z_{0}\left(x_{0}-b y_{0}\right)\right),
\end{aligned}
$$




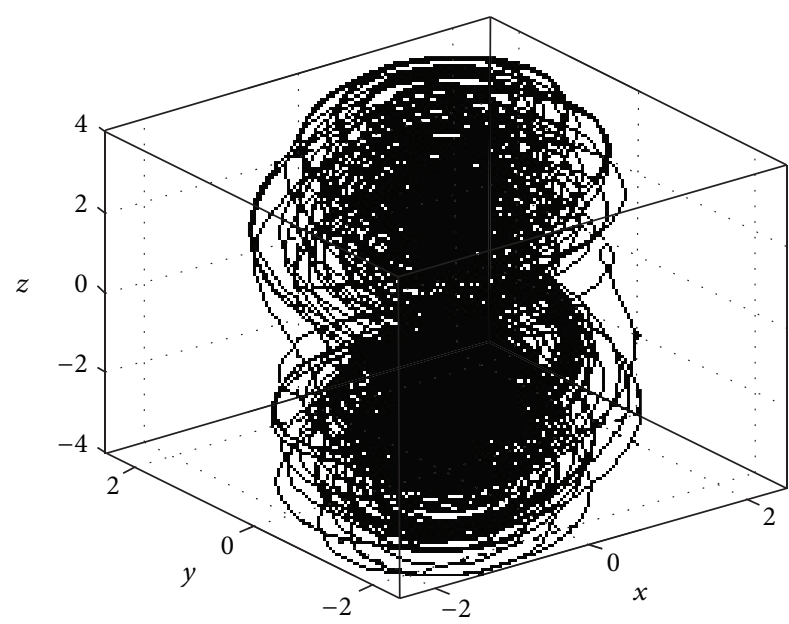

(a)

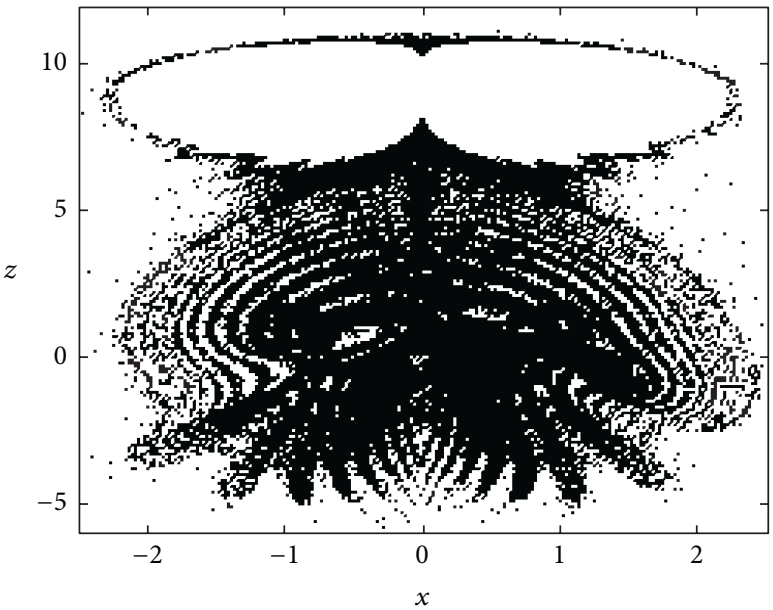

(b)

Figure 2: (a) Time history and (b) Poincaré map in $x-z\{y=0\}$ plane of chaotic system (7).

$$
\begin{aligned}
y_{1}(t) & =y_{0}+I^{\alpha}\left(z_{0}\left(b x_{0}-c y_{0}\right)\right) \\
& =y_{0}+\frac{t^{\alpha}}{\alpha \Gamma(\alpha)}\left(z_{0}\left(b x_{0}-c y_{0}\right)\right), \\
z_{1}(t) & =z_{0}+I^{\alpha}\left(1-x_{0}^{2}-y_{0}^{2}\right) \\
& =z_{0}+\frac{t^{\alpha}}{\alpha \Gamma(\alpha)}\left(1-x_{0}^{2}-y_{0}^{2}\right) .
\end{aligned}
$$

Second, let $t \in[s, 2 s)$, so $t / s \in[1,2)$. Hence, we get

$$
\begin{aligned}
& D^{\alpha} x(t)=a+z_{1}\left(x_{1}-b y_{1}\right), \\
& D^{\alpha} y(t)=z_{1}\left(b x_{1}-c y_{1}\right), \\
& D^{\alpha} z(t)=1-x_{1}^{2}-y_{1}^{2},
\end{aligned}
$$

which have the following solutions:

$$
\begin{aligned}
x_{2}(t)= & x_{1}(s)+I^{\alpha}\left(a+z_{1}(s)\left(x_{1}(s)-b y_{1}(s)\right)\right) \\
= & x_{1}(s) \\
& +\frac{(t-s)^{\alpha}}{\alpha \Gamma(\alpha)}\left(a+z_{1}(s)\left(x_{1}(s)-b y_{1}(s)\right)\right), \\
y_{2}(t)= & y_{1}(s)+I^{\alpha}\left(z_{1}(s)\left(b x_{1}(s)-c y_{1}(s)\right)\right) \\
= & y_{1}(s)+\frac{(t-s)^{\alpha}}{\alpha \Gamma(\alpha)}\left(z_{1}(s)\left(b x_{1}(s)-c y_{1}(s)\right)\right), \\
z_{2}(t)= & z_{1}(s)+I^{\alpha}\left(1-x_{1}^{2}(s)-y_{1}^{2}(s)\right) \\
= & z_{1}(s)+\frac{(t-s)^{\alpha}}{\alpha \Gamma(\alpha)}\left(1-x_{1}^{2}(s)-y_{1}^{2}(s)\right) .
\end{aligned}
$$

Thus, after repeating the discretization process $n$ times, we obtain

$$
\begin{aligned}
x_{n+1}( & t) \\
= & x_{n}(n s)+I^{\alpha}\left(a+z_{n}(n s)\left(x_{n}(n s)-b y_{n}(n s)\right)\right) \\
= & x_{n}(n s) \\
& \quad+\frac{(t-n s)^{\alpha}}{\alpha \Gamma(\alpha)}\left(a+z_{n}(n s)\left(x_{n}(n s)-b y_{n}(n s)\right)\right), \\
y_{n+1}( & (t)=y_{n}(n s)+I^{\alpha}\left(z_{n}(n s)\left(b x_{n}(n s)-c y_{n}(n s)\right)\right) \\
= & y_{n}(n s) \\
& +\frac{(t-n s)^{\alpha}}{\alpha \Gamma(\alpha)}\left(z_{n}(n s)\left(b x_{n}(n s)-c y_{n}(n s)\right)\right), \\
z_{n+1}( & t)=z_{n}(n s)+I^{\alpha}\left(1-x_{n}^{2}(n s)-y_{n}^{2}(n s)\right) \\
= & z_{n}(n s)+\frac{(t-n s)^{\alpha}}{\alpha \Gamma(\alpha)}\left(1-x_{n}^{2}(n s)-y_{n}^{2}(n s)\right),
\end{aligned}
$$

where $t \in[n s,(n+1) s)$. For $t \rightarrow(n+1) s$, system (14) is reduced to

$$
\begin{aligned}
x_{n+1} & =x_{n}+I^{\alpha}\left(a+z_{n}\left(x_{n}-b y_{n}\right)\right) \\
& =x_{n}+\frac{s^{\alpha}}{\alpha \Gamma(\alpha)}\left(a+z_{n}\left(x_{n}-b y_{n}\right)\right), \\
y_{n+1} & =y_{n}+I^{\alpha}\left(z_{n}\left(b x_{n}-c y_{n}\right)\right) \\
& =y_{n}+\frac{s^{\alpha}}{\alpha \Gamma(\alpha)}\left(z_{n}\left(b x_{n}-c y_{n}\right)\right), \\
z_{n+1} & =z_{n}+I^{\alpha}\left(1-x_{n}^{2}-y_{n}^{2}\right) \\
& =z_{n}+\frac{s^{\alpha}}{\alpha \Gamma(\alpha)}\left(1-x_{n}^{2}-y_{n}^{2}\right) .
\end{aligned}
$$




\section{Dynamical Behaviors of the Fractional- Order Semiconductor Lasers Model}

4.1. Existence and Uniqueness of the Solution. The fractional order of the modified optically injected semiconductor lasers system can be written as

$$
D^{\alpha} X(t)=F(X(t)), \quad t \in(0, T], X(0)=X_{0},
$$

where

$$
\begin{aligned}
X & =\left[\begin{array}{l}
x \\
y \\
z
\end{array}\right], \\
X_{0} & =\left[\begin{array}{l}
x_{0} \\
y_{0} \\
z_{0}
\end{array}\right], \\
F(X) & =\left[\begin{array}{c}
a+z(x-b y) \\
z(b x-c y) \\
1-x^{2}-y^{2}
\end{array}\right] .
\end{aligned}
$$

Define the supremum norm as

$$
\|N\|=\sup _{t \in(0, T]}|N(t)| ;
$$

then, the norm of the matrix $M=\left[m_{i j}[t]\right]$ is defined by

$$
\|M\|=\sum_{i, j} \sup _{t \in(0, T]}\left|m_{i j}[t]\right| .
$$

We investigate the existence and uniqueness of the solution in the region $\Omega \times(0, T]$, where

$$
\Omega=\{(x, y, z): \max (|x|,|y|,|z|) \leq \xi\} .
$$

Hence, we can get the solution of system (8) as follows:

$$
X=X_{0}+\frac{1}{\Gamma(\alpha)} \int_{0}^{t}(t-\tau)^{\alpha-1} F(X(\tau)) d \tau,
$$

so

$$
\begin{aligned}
H & \left(X_{1}\right)-H\left(X_{2}\right) \\
& =\frac{1}{\Gamma(\alpha)} \int_{0}^{t}(t-\tau)^{\alpha-1}\left(F\left(X_{1}(\tau)\right)-F\left(X_{2}(\tau)\right)\right) d \tau .
\end{aligned}
$$

Thus, we get the following inequality:

$$
\begin{aligned}
& \left|H\left(X_{1}\right)-H\left(X_{2}\right)\right| \\
& \quad \leq \frac{1}{\Gamma(\alpha)} \int_{0}^{t}\left|(t-\tau)^{\alpha-1}\left(F\left(X_{1}(\tau)\right)-F\left(X_{2}(\tau)\right)\right)\right| d \tau,
\end{aligned}
$$

which yields

$$
\begin{aligned}
& \left\|H\left(X_{1}\right)-H\left(X_{2}\right)\right\| \\
& \quad \leq \frac{T^{\alpha}}{\Gamma(\alpha+1)} \max \left\{-\xi e^{-\xi}, c \xi e^{-c \xi}, 0\right\}\left\|X_{1}-X_{2}\right\|,
\end{aligned}
$$

where

$$
L=\frac{T^{\alpha}}{\Gamma(\alpha+1)} \max \left\{-\xi e^{-\xi}, c \xi e^{-c \xi}, 0\right\} ;
$$

the mapping $X=H(X)$ is a contraction mapping if $L<1$, and the following theorem gives the sufficient condition for existence and uniqueness of the solution of system (8).

Theorem 2. The sufficient condition for existence and uniqueness of the solution of system (8) in the region $\Omega \times(0, T]$ with initial conditions $X(0)=X_{0}$ and $t \in(0, T]$ is

$$
L=\frac{T^{\alpha}}{\Gamma(\alpha+1)} \max \left\{-\xi e^{-\xi}, c \xi e^{-c \xi}, 0\right\}<1 .
$$

4.2. Stability of the Fixed Points of the Discrete System. In order to find the equilibrium points of system (8), we let

$$
\begin{aligned}
& \frac{d^{\alpha} x}{d t^{\alpha}}=0, \\
& \frac{d^{\alpha} y}{d t^{\alpha}}=0, \\
& \frac{d^{\alpha} z}{d t^{\alpha}}=0,
\end{aligned}
$$

and from direct calculation we know that the system has at most five nonnegative equilibrium points:

$$
\begin{aligned}
& E_{0}=\left(\frac{c}{\sqrt{c^{2}+b^{2}}}, \frac{b}{\sqrt{c^{2}+b^{2}}}, \frac{a \sqrt{c^{2}+b^{2}}}{b^{2}-c}\right), \\
& E_{1}=\left(-\frac{c}{\sqrt{c^{2}+b^{2}}},-\frac{b}{\sqrt{c^{2}+b^{2}}},-\frac{a \sqrt{c^{2}+b^{2}}}{b^{2}-c}\right) .
\end{aligned}
$$

By considering the Jacobian matrices for these fixed points and calculating their eigenvalues, we can investigate the local stability of these points based on the roots of the model's characteristic equation [42]. The Jacobian matrix of system (15) is given by

$$
\begin{aligned}
& J\left(x_{n}, y_{n}, z_{n}\right) \\
& =\left(\begin{array}{ccc}
1+\frac{s^{\alpha} z_{n}}{\alpha \Gamma(\alpha)} & -\frac{s^{\alpha} b z_{n}}{\alpha \Gamma(\alpha)} & \frac{s^{\alpha}\left(x_{n}-b y_{n}\right)}{\alpha \Gamma(\alpha)} \\
\frac{s^{\alpha} b z_{n}}{\alpha \Gamma(\alpha)} & 1-\frac{s^{\alpha} c z_{n}}{\alpha \Gamma(\alpha)} & \frac{s^{\alpha}\left(b x_{n}-c y_{n}\right)}{\alpha \Gamma(\alpha)} \\
-\frac{2 s^{\alpha} x_{n}}{\alpha \Gamma(\alpha)} & -\frac{2 s^{\alpha} y_{n}}{\alpha \Gamma(\alpha)} & 1
\end{array}\right) .
\end{aligned}
$$

Theorem 3. For system (15), the following statements hold true:

(i) $E_{0}$ is unstable if ac $>0$.

(ii) $E_{0}$ is locally asymptotically stable if and only if $0<a<$ $\alpha \Gamma(\alpha) / s^{\alpha}-2 s^{\alpha} / \alpha \Gamma(\alpha)$ 
Proof. The Jacobian matrix evaluated at the fixed point $E_{0}$ is

$$
J\left(E_{0}\right)=\left(\begin{array}{ccc}
1-\frac{s^{\alpha} a}{\alpha \Gamma(\alpha)} & 0 & \frac{s^{\alpha}}{\alpha \Gamma(\alpha)} \\
0 & 1+\frac{s^{\alpha} c a}{\alpha \Gamma(\alpha)} & 0 \\
\frac{2 s^{\alpha}}{\alpha \Gamma(\alpha)} & 0 & 1
\end{array}\right)
$$

The eigenvalues of $J\left(E_{0}\right)$ are $\lambda_{2}=1+s^{\alpha} c a / \alpha \Gamma(\alpha)$ and $\lambda_{1,3}=$ $1-s^{\alpha} a / 2 \alpha \Gamma(\alpha) \pm\left(s^{\alpha} / 2 \alpha \Gamma(\alpha)\right) \sqrt{a^{2}+8}$. Since $0<\alpha \leq 1$ and $s>0, s^{\alpha} / \alpha \Gamma(\alpha), a c>0$, then $\lambda_{2}>1$ which implies that the fixed point $E_{0}$ is unstable. If $0<a<\alpha \Gamma(\alpha) / s^{\alpha}-$ $2 s^{\alpha} / \alpha \Gamma(\alpha)$, the eigenvalues of $J\left(E_{0}\right)$ are $\lambda_{1,3}=1-s^{\alpha} a / 2 \alpha \Gamma(\alpha) \pm$ $\left(s^{\alpha} / 2 \alpha \Gamma(\alpha)\right) \sqrt{a^{2}+8}<1$ and $\lambda_{2}=1+s^{\alpha} c a / \alpha \Gamma(\alpha)<1$, so the stability conditions $\left|\lambda_{2}\right|<1$ and $\left|\lambda_{1,3}\right|<1$ are satisfied if $0<a<\alpha \Gamma(\alpha) / s^{\alpha}-2 s^{\alpha} / \alpha \Gamma(\alpha)$.

\subsubsection{The Case $b=0$}

Theorem 4. For system (15), the following statements hold true:

(i) $E_{1}$ is unstable if ac $<0$.

(ii) $E_{1}$ is locally asymptotically stable if and only if $a<$ $-2 \sqrt{2}$.

Proof. The Jacobian matrix evaluated at the fixed point $E_{1}$ is

$$
J\left(E_{1}\right)=\left(\begin{array}{ccc}
1+\frac{s^{\alpha} a}{\alpha \Gamma(\alpha)} & 0 & -\frac{s^{\alpha}}{\alpha \Gamma(\alpha)} \\
0 & 1-\frac{s^{\alpha} c a}{\alpha \Gamma(\alpha)} & 0 \\
\frac{2 s^{\alpha}}{\alpha \Gamma(\alpha)} & 0 & 1
\end{array}\right)
$$

The eigenvalues of $J\left(E_{1}\right)$ are $\lambda_{2}=1-s^{\alpha} c a / \alpha \Gamma(\alpha)$ and $\lambda_{1,3}=$ $1+s^{\alpha} a / 2 \alpha \Gamma(\alpha) \pm\left(s^{\alpha} / 2 \alpha \Gamma(\alpha)\right) \sqrt{a^{2}-8}$. Since $0<\alpha \leq 1$ and $s>0, s^{\alpha} / \alpha \Gamma(\alpha), a c<0$, then $\lambda_{2}>1$ which implies that the fixed point $E_{0}$ is unstable. If $a<-2 \sqrt{2}$, the eigenvalues of $J\left(E_{1}\right)$ are $\lambda_{2}=1+s^{\alpha} c a / \alpha \Gamma(\alpha)<1$ and $\lambda_{1,3}=1+s^{\alpha} a / 2 \alpha \Gamma(\alpha) \pm$ $\left(s^{\alpha} / 2 \alpha \Gamma(\alpha)\right) \sqrt{a^{2}-8}<1$, so the stability conditions $\left|\lambda_{2}\right|<1$ and $\left|\lambda_{1,3}\right|<1$ are satisfied if $a<-2 \sqrt{2}$.

4.2.2. The Case $b \neq 0$. Next, we will discuss the stability of the fixed point $E_{1}$. The Jacobian matrix of system (15) evaluated at $E_{1}$ is

$$
J\left(E_{1}\right)=\left(\begin{array}{lll}
\gamma_{11} & \gamma_{12} & \gamma_{13} \\
\gamma_{21} & \gamma_{22} & \gamma_{23} \\
\gamma_{31} & \gamma_{32} & \gamma_{33}
\end{array}\right)
$$

where

$$
\begin{aligned}
& \gamma_{11}=1+\frac{s^{\alpha} a \sqrt{c^{2}+b^{2}}}{\alpha\left(b^{2}-c\right) \Gamma(\alpha)}, \\
& \gamma_{12}=-\frac{s^{\alpha} b a \sqrt{c^{2}+b^{2}}}{\alpha\left(b^{2}-c\right) \Gamma(\alpha)}, \\
& \gamma_{13}=-\frac{s^{\alpha}\left(b^{2}-c\right)}{\alpha \Gamma(\alpha) \sqrt{c^{2}+b^{2}}}, \\
& \gamma_{21}=\frac{s^{\alpha} b \sqrt{c^{2}+b^{2}}}{\alpha\left(b^{2}-c\right) \Gamma(\alpha)}, \\
& \gamma_{22}=1-\frac{s^{\alpha} c a \sqrt{c^{2}+b^{2}}}{\alpha\left(b^{2}-c\right) \Gamma(\alpha)}, \\
& \gamma_{23}=0, \\
& \gamma_{31}=\frac{-2 s^{\alpha} c}{\alpha \sqrt{c^{2}+b^{2}} \Gamma(\alpha)}, \\
& \gamma_{32}=\frac{-2 s^{\alpha} b}{\alpha \sqrt{c^{2}+b^{2}} \Gamma(\alpha)}, \\
& 1 .
\end{aligned}
$$

The characteristic equation of $E_{1}$ is given by

$$
\lambda^{3}+A_{1} \lambda^{2}+A_{2} \lambda+A_{3}=0
$$

where

$$
\begin{aligned}
& A_{1}=2-V_{1}, \\
& A_{2}=-3+V_{2}-V_{3}-V_{4}+V_{5}, \\
& A_{3}=-1-V_{6}+V_{2}-V_{7}-V_{8}, \\
& V_{1}=\frac{s^{\alpha} c a \sqrt{c^{2}+b^{2}}}{\alpha\left(b^{2}-c\right) \Gamma(\alpha)}, \\
& V_{2}=\frac{2 s^{2 \alpha} c\left(b^{2}-c\right)}{\alpha^{2} \Gamma^{2}(\alpha)\left(c^{2}+b^{2}\right)}, \\
& V_{3}=\frac{s^{\alpha} a \sqrt{c^{2}+b^{2}}}{\alpha\left(b^{2}-c\right) \Gamma(\alpha)}, \\
& V_{4}=\frac{s^{2 \alpha} b^{2} a\left(c^{2}+b^{2}\right)}{\alpha^{2}\left(b^{2}-c\right)^{2} \Gamma^{2}(\alpha)}, \\
& V_{5}=\frac{s^{2 \alpha} c a^{2}\left(c^{2}+b^{2}\right)}{\alpha^{2}\left(b^{2}-c\right)^{2} \Gamma^{2}(\alpha)}, \\
& V_{6}=\frac{-2 s^{3 \alpha}\left(b^{2}+2 a c^{2}\right)}{\alpha^{3} \sqrt{c^{2}+b^{2}} \Gamma^{3}(\alpha)},
\end{aligned}
$$




$$
\begin{aligned}
& V_{7}=\frac{s^{2 \alpha}\left(b^{2}-a c\right) a\left(c^{2}+b^{2}\right)}{\alpha^{2}\left(b^{2}-c\right)^{2} \Gamma^{2}(\alpha)}, \\
& V_{8}=\frac{s^{\alpha}(1-c) a \sqrt{c^{2}+b^{2}}}{\alpha\left(b^{2}-c\right) \Gamma(\alpha)} .
\end{aligned}
$$

According to Jury's criterion [42], the fixed point $E_{1}$ is locally asymptotically stable if

$$
\begin{array}{r}
1+A_{1}+A_{2}+A_{3}>0, \\
1-A_{1}+A_{2}-A_{3}>0, \\
1-A_{2}+A_{1} A_{3}-A_{3}^{2}>0, \\
1+A_{2}-A_{1} A_{3}-A_{3}^{2}>0 .
\end{array}
$$

Thus, conditions (36) imply that the fixed point $E_{0}$ is locally asymptotically stable if the system's parameters belong to the set

$$
\begin{aligned}
& \left\{(a, b, c, \alpha, s):-\left[1+V_{6}-V_{2}+V_{7}+V_{8}\right] \in \Omega_{i} \forall i\right. \\
& \quad=1,2,3\},
\end{aligned}
$$

where

$$
\begin{aligned}
\Omega_{1} & =\left[V_{1}-V_{2}+V_{3}+V_{4}-V_{5},-4+V_{1}+V_{2}-V_{3}\right. \\
& \left.-V_{4}+V_{5}\right], \\
\Omega_{2} & =\left[2-V_{1}\right. \\
& -\frac{\sqrt{\left(2-V_{1}\right)^{2}-4\left(-4+V_{2}-V_{3}-V_{4}+V_{5}\right)}}{2}, 2 \\
& \left.-V_{1}-\frac{\sqrt{\left(2-V_{1}\right)^{2}-4\left(-4+V_{2}-V_{3}-V_{4}+V_{5}\right)}}{2}\right], \\
\Omega_{3} & =\left[-2+V_{1}\right. \\
& \left.+V_{1}+\frac{\sqrt{\left(2-V_{1}\right)^{2}-4\left(-4+V_{2}-V_{3}-V_{4}+V_{5}\right)}}{2}\right] . \\
& -\frac{\sqrt{\left(2-V_{1}\right)^{2}-4\left(-4+V_{2}-V_{3}-V_{4}+V_{5}\right)}}{2},
\end{aligned}
$$

Lemma 5 (an explicit criterion of Hopf bifurcation in maps [43]). Let $X^{*}$ be a fixed point of the nth-order discrete-time dynamical system; the characteristic equation of the Jacobian matrix $A=\left(a_{i j}\right)_{n \times n}$ is given as

$$
P_{v}(\lambda)=\lambda^{n}+s_{1} \lambda^{n-1}+\cdots+s_{n-1} \lambda+s_{n}
$$

where $s_{j}=s_{j}(v, k), j=1, \ldots, n, v$ is the bifurcation parameter, and $k$ is the control parameter or another parameter to be determined. Consider the sequence of determinants $\Delta_{0}^{ \pm}(v, k)=$ $1, \Delta_{1}^{ \pm}(v, k), \ldots, \Delta_{n}^{ \pm}(v, k)$, where

$$
\begin{gathered}
\Delta_{j}^{ \pm}(v, k)=\mid\left(\begin{array}{ccccc}
1 & s_{1} & s_{2} & \cdots & s_{j-1} \\
0 & 1 & s_{1} & \cdots & s_{j-2} \\
0 & 0 & 1 & \cdots & s_{j-3} \\
\vdots & \vdots & \vdots & \cdots & \vdots \\
0 & 0 & 0 & \cdots & 1
\end{array}\right) \\
\pm\left(\begin{array}{ccccc}
s_{n-j+1} & s_{n-j+2} & \cdots & s_{n-1} & s_{n} \\
s_{n-j+1} & s_{n-j+3} & \cdots & s_{n} & 0 \\
\vdots & \vdots & \cdots & \vdots & \vdots \\
s_{n-1} & s_{n} & \cdots & 0 & 0 \\
s_{n} & 0 & \cdots & 0 & 0
\end{array}\right) \mid,
\end{gathered}
$$

However, the following conditions hold:

(H1) Eigenvalue assignment $\Delta_{n-1}^{-}\left(v_{0}, k\right)=0, P_{v_{0}}(1)>0$, $(-1)^{n} P_{v_{0}}(-1)>0, \Delta_{n-1}^{-}\left(v_{0}, k\right)>0, \Delta_{n-1}^{+}\left(v_{0}, k\right)=0$, $\Delta_{j}^{ \pm}\left(v_{0}, k\right)>0, j=n-3, n-5, \ldots, 1$ (or 2) when $n$ is even (or odd, resp.).

(H2) Transversality condition $d \Delta_{n-1}^{-}\left(v_{0}, k\right) / d v \neq 0$.

(H3) Nonresonance condition $\cos (2 \pi / m) \neq \varphi$ or resonance condition $\cos (2 \pi / m)=\varphi$, where $m=3,4,5, \ldots$ and $\varphi=1-0.5 P_{v_{0}}(1) \Delta_{n-3}^{-}\left(v_{0}, k\right) / \Delta_{n-2}^{+}\left(v_{0}, k\right)$; then Hopf bifurcation occurs at $v_{0}$.

\section{Numerical Simulations}

Even if it is possible to obtain the analytical conditions, the processing is very difficult. So, in order to analyze the stability of the interior fixed points, we investigate the global dynamical behavior of system (15) through numerical simulations. And we show some attractors of system (15), such as asymptotic behaviors near the equilibrium points, periodic orbits, and strange attractors. In the following, we will use the numerical simulations to show the influence of different fractional-order parameters $q$ and $s$ on the stability of system (15)

We fixed $a=0.4$ and $b=3$ and vary the parameters $q, s$, and $c$. Let $s=0.01$. Figures 3(a)-3(c) show the portraits of system (15) with $q=0.87, q=0.93$, and $q=$ 0.98 , respectively. The numerical results show that with the increase of fractional-order parameter $q$ the discrete system (15) is consistent with the fractional-order system (8).

When we fixed $s=0.01$, Figures 3(d)-3(f) depict the attractors of system (15) with $q=0.7, q=0.8$, and $q=$ 0.87 , respectively. And when $s=0.001$, we present the phase portraits of system (15) with $q=0.9, q=0.93$, and $q=0.97$, as shown in Figures 3(g)-3(i), respectively. From 


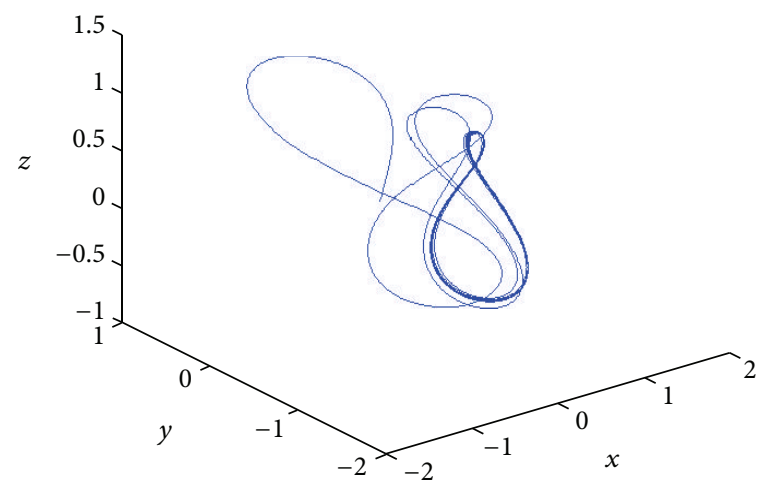

(a) $c=-0.1, q=0.87$, and $s=0.01$

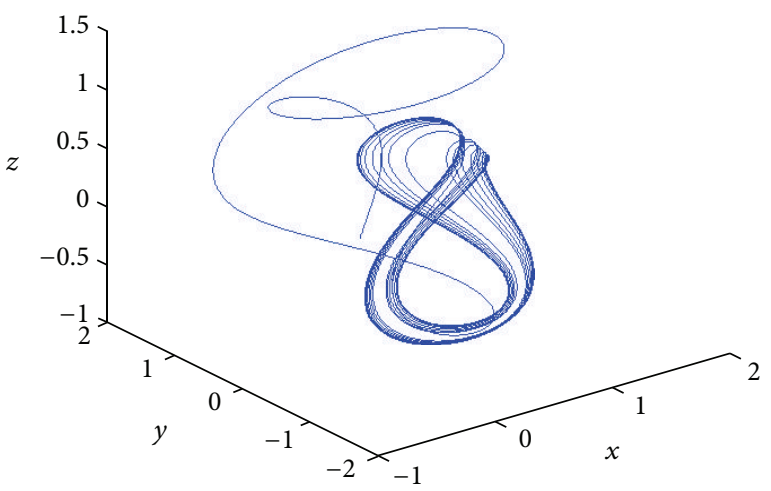

(c) $c=-0.8, q=0.98$, and $s=0.01$

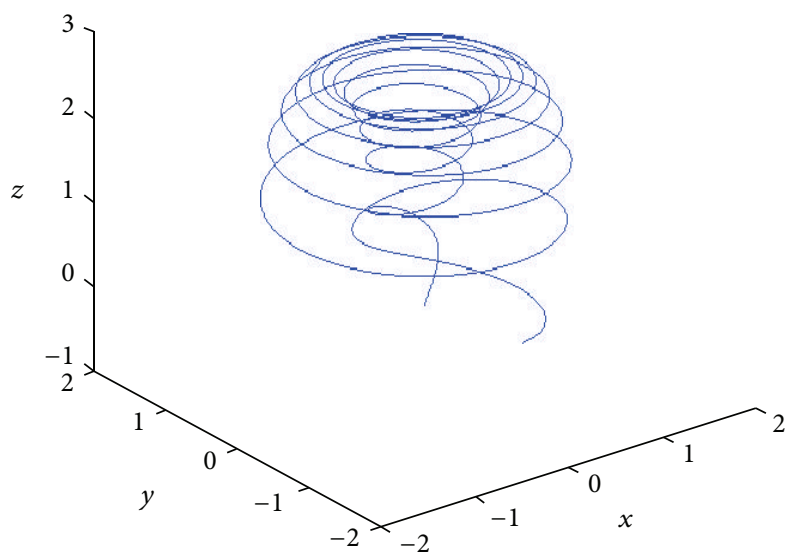

(e) $c=-1, q=0.8$, and $s=0.01$

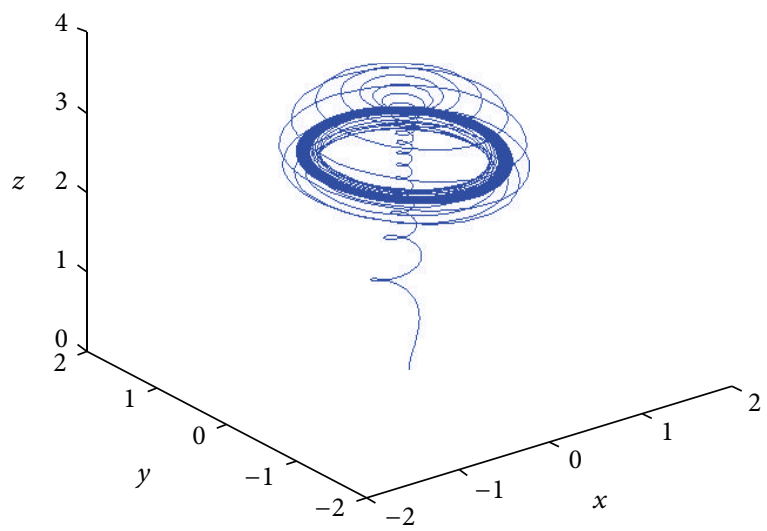

(g) $c=-0.8, q=0.9$, and $s=0.001$

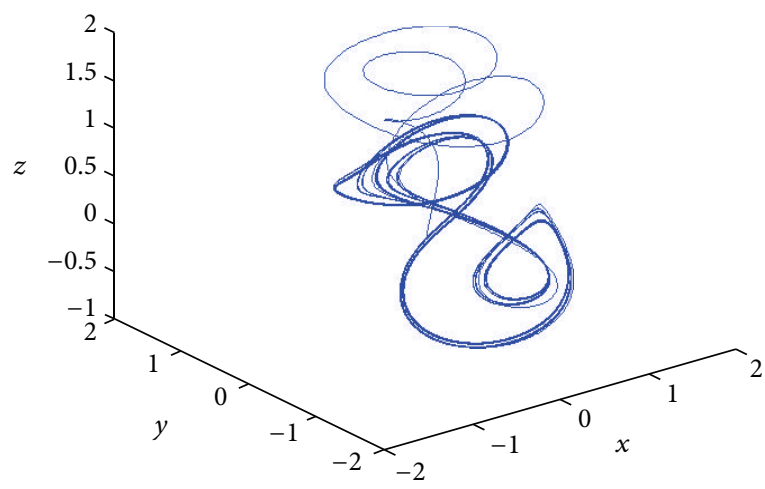

(b) $c=-1.7, q=0.93$, and $s=0.01$

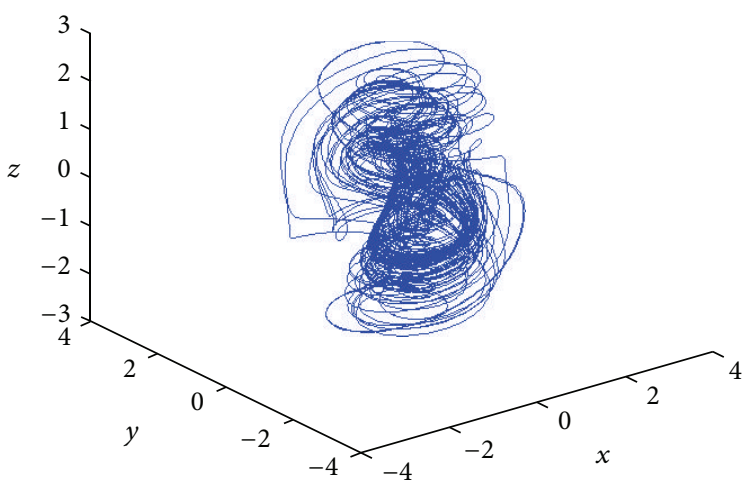

(d) $c=-1.9, q=0.7$, and $s=0.01$

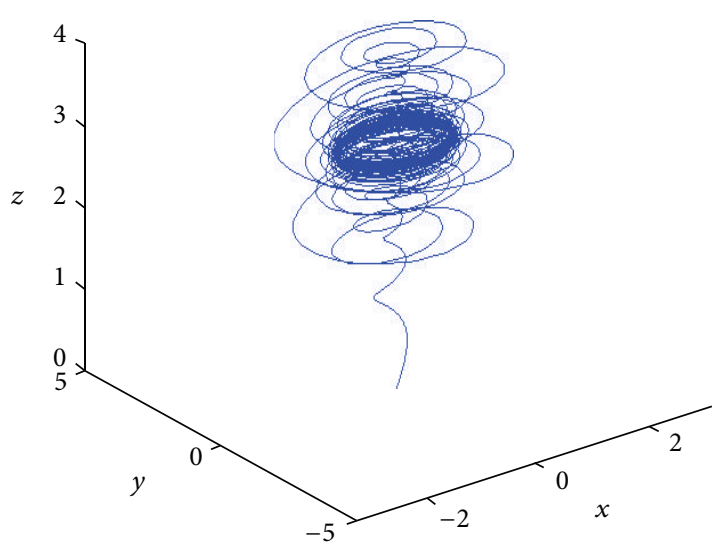

(f) $c=-0.1, q=0.87$, and $s=0.01$

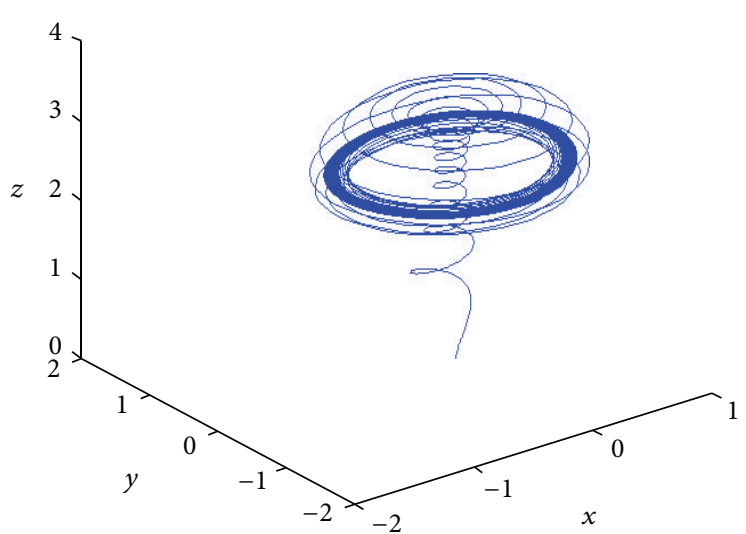

(h) $c=-0.87, q=0.93$, and $s=0.001$

FIgUre 3: Continued. 


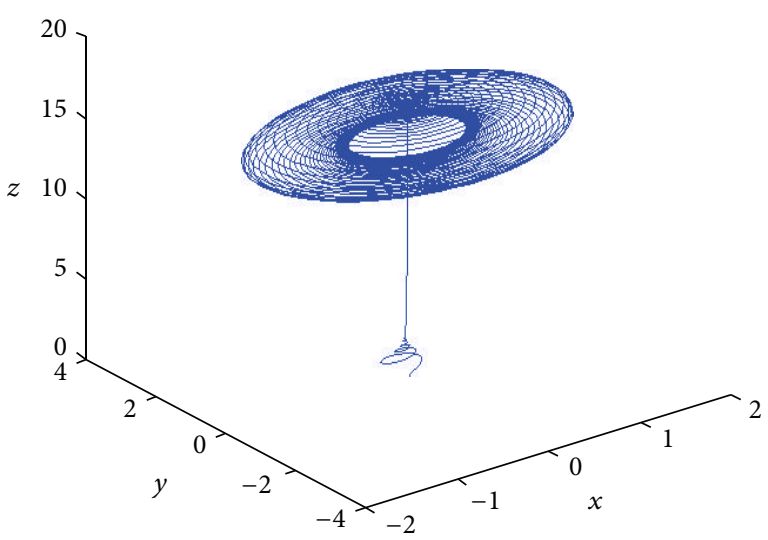

(i) $c=-0.1, q=0.97$, and $s=0.001$

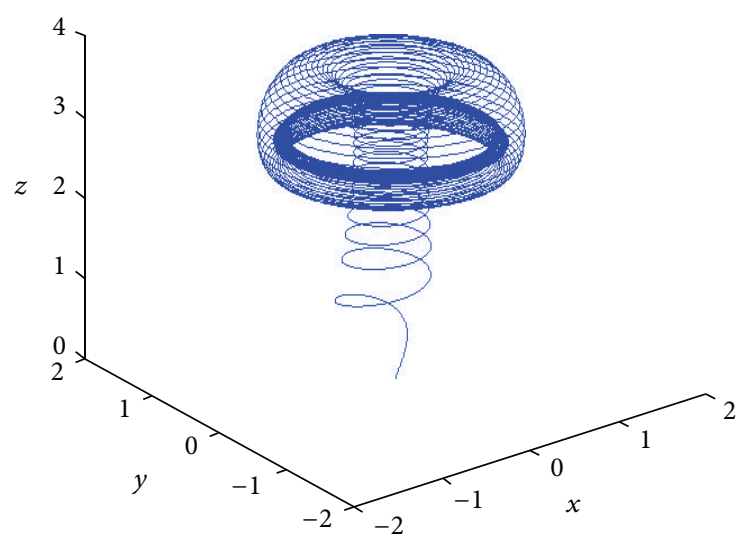

(j) $c=-1.9, q=0.98$, and $s=0.001$

FIGURE 3: Trajectory of system (15) in phase space when $a=0.4$ and $b=3$.

Figures 3(d)-3(i), we notice that the chaotic behavior of system (15) gradually becomes stable with the reduction of the parameter $s$ and the gradual increase in the fractional-order parameter $q$.

Figure $3(\mathrm{j})$ is the strange attractor of system (15) with $q=0.98$ and $s=0.001$. Obviously, it confirms the above result; this is because Figure 3(j) shows that the reversibility of the above result, namely, the stable behavior of system (15), is destabilizing when $s$ is small and decreasing the fractionalorder parameter $q$.

We let the time series length be equal to 3000 . Figure 4(a) shows the curve of $\log C(r, m)-\log r$ when the parameter $m$ varies in $[1,18]$, and the error bars are purely statistical. The curves attain a constant slope (the same for all curves) as $r \rightarrow 0$ and the slope should be equal to the information dimension. The slope curve of the time serials is shown in Figure 4(b). In Figure 4(c), with the various embedding dimension, correlation exponent is on the increase. When $m \geq 8$, the sharp increase to very big values indicates that $D_{2}=1.1466$. When embedding dimension increases to 7 , the curve above it appears to converge and we compute the Kolmogorov entropy $K=0.3096$. From Figure 4(d), we found that the curves appear to converge to this curve at least up to the accuracy that we could hope to achieve in such numerical experiments. In general, results were found to be more sensitive to the number of iterations than to the averaging number.

From the above numerical experiments, we get the following results:

(1) When embedding dimension varies from 6 to 8 , the correlation integral begins to gradually saturate. A correlation exponent of the different system verified by the number of the time series needs specific analysis.

(2) If embedding dimension does not exceed the saturated values of the minimum embedding dimension, then the estimation of the correlation exponents is not up to the precision with which we could hope to extract the metric entropy.
(3) The constancy of the slopes will break down for $r$ smaller than some value $r_{0}$. With the incensement of the embedding dimension, the distances between points increase, and $r_{0}$ shifts to higher values of $r$. When the embedding dimension is to achieve a certain value, the Kolmogorov entropy reaches saturation and begins to converge.

However, when the bifurcation diagram loses its continuity, this means that the state of the system is either quasiperiodic or chaotic, as shown in Figures 5(a) and 5(b). And, according to Lemma 5, we select $a=0.4, b=3, q=0.98$, and $s=0.01$ and draw the phase diagram of system (15) with $c=-0.8, c=-1$, and $c=-1.9$, as shown in Figures 6-8, respectively. From Figures $6-8$, we know that system (15) loses its stability with the gradual decrease of bifurcation parameter c. That is to say, by choosing an appropriate bifurcation parameter, we can prove that the Hopf bifurcation occurs when the bifurcation parameter passes through the critical value.

\section{Conclusion}

In this paper, we introduced a fractional order of modified optically injected semiconductor lasers model and discretized this system by using a new discretization technique. More precisely, a sufficient condition for existence and uniqueness of the solution of the proposed fractional-order system is investigated, and we also studied the local stability of the equilibrium of the discrete fractional-order semiconductor lasers system. Moreover, the results showed that the fractional parameter $q$ has an effect on the stability of the discrete system. And the chaotic behavior of system (15) will be stabilized when reducing the value of parameter $s$ and increasing the fractional-order parameter $q$. Meanwhile, the stable behavior of system (15) is destabilizing when decreasing the fractional-order parameter $q$. We also found that the discrete system exhibits much richer dynamical behaviors than its corresponding fractional-order counterpart, and the existence of Hopf bifurcation of discrete system has been 


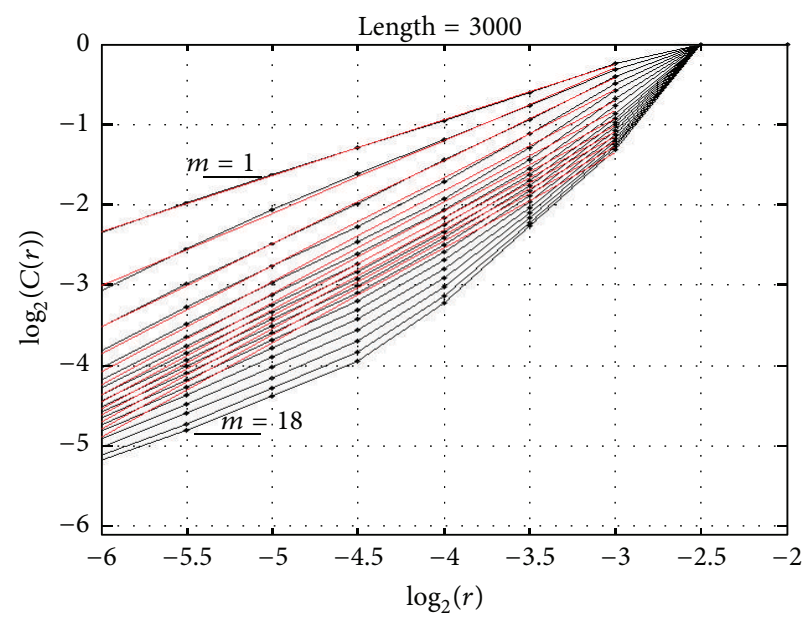

(a)

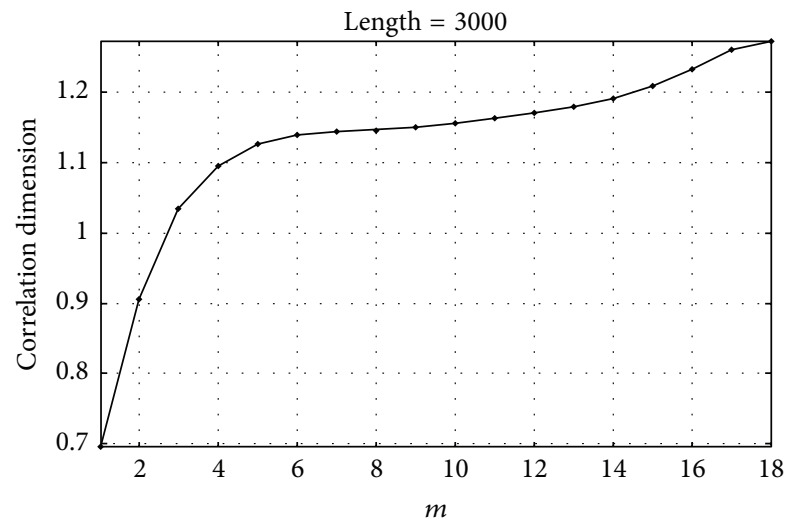

(c)

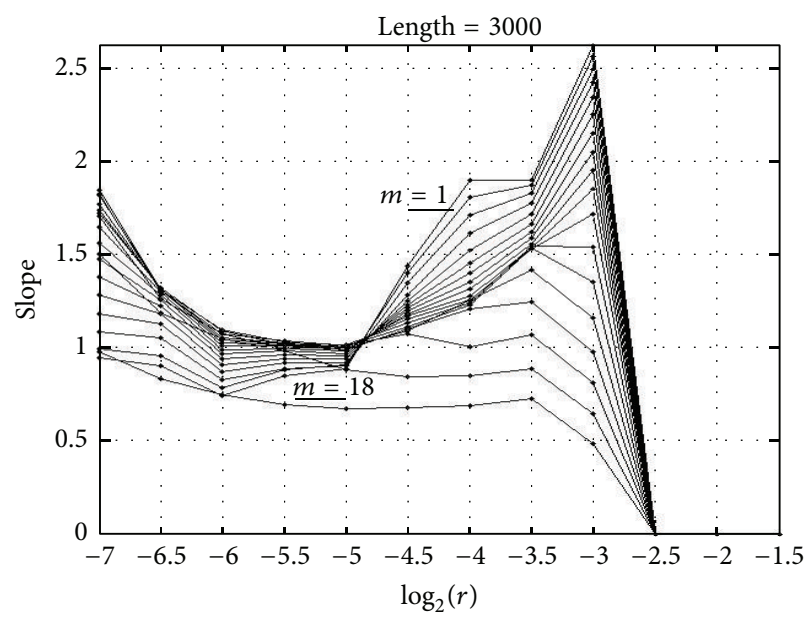

(b)

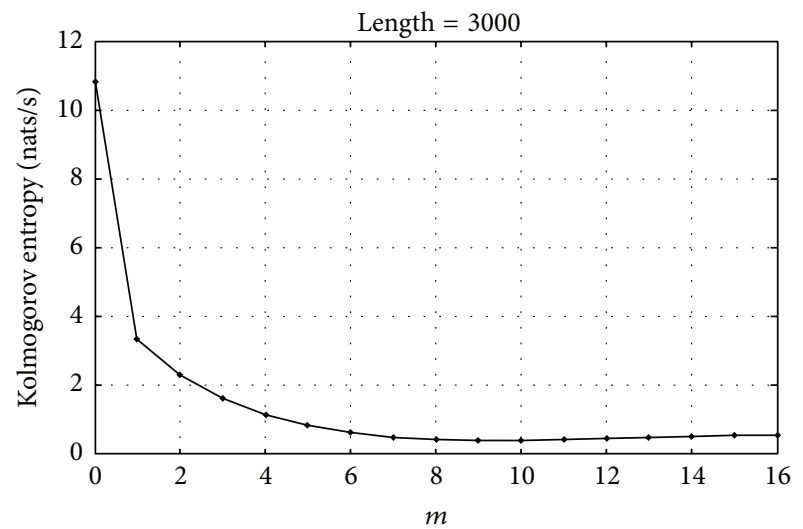

(d)

Figure 4: At $N=3000$. (a) Correlation integral curve. Points pertaining to the same value of $m$ are connected by lines. The values of $m$ are $m=1$ (top curve), 4, $6, \ldots, 18$ (bottom curve); (b) slope curve of the time serials. An ordinate value is the slope of $\log C(r, m)$ and $\log r$; (c) correlation exponent curve $D_{2}=1.1466$; (d) the Kolmogorov entropy of system (15) attractor is equal to $D_{2}=0.3096$.

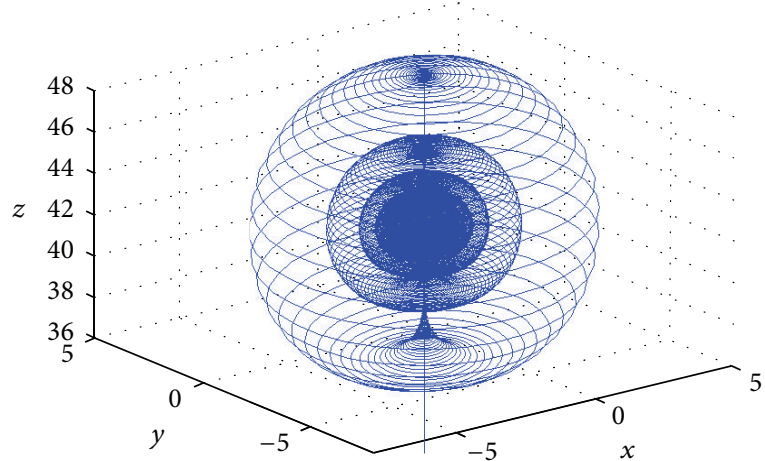

(a)

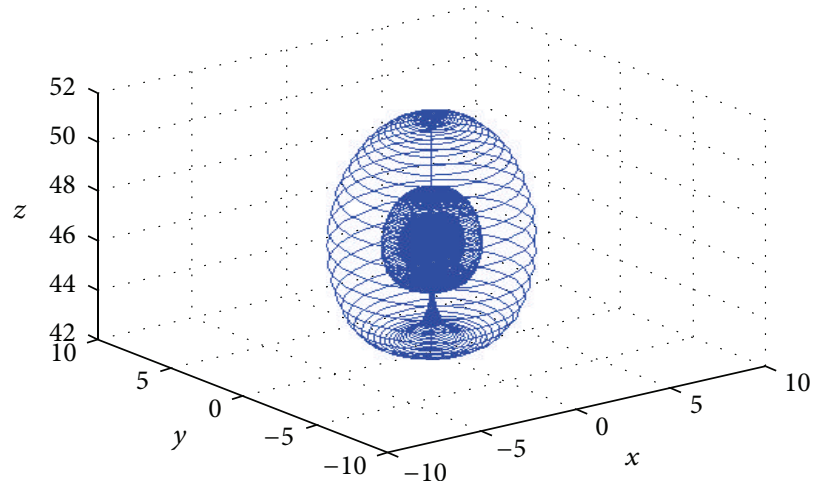

(b)

FIgURE 5: (a) Phase trajectory in 3D space with $a=0.4, b=3, c=-1.9, q=0.96$, and $s=0.001$; (b) interior phase trajectory of (a).

verified. Finally, the numerical simulations are given to show the chaotic attractors of the fractional-order system and the richer dynamics of its discrete counterpart. In addition, we proposed a method to estimate the Kolmogorov entropy and use it to verify the state of the system. Future work on the topic may be extended to apply the chaos control (OGY method, feedback control method, and adaptive control method) or bifurcation control (the linear and nonlinear feedback 


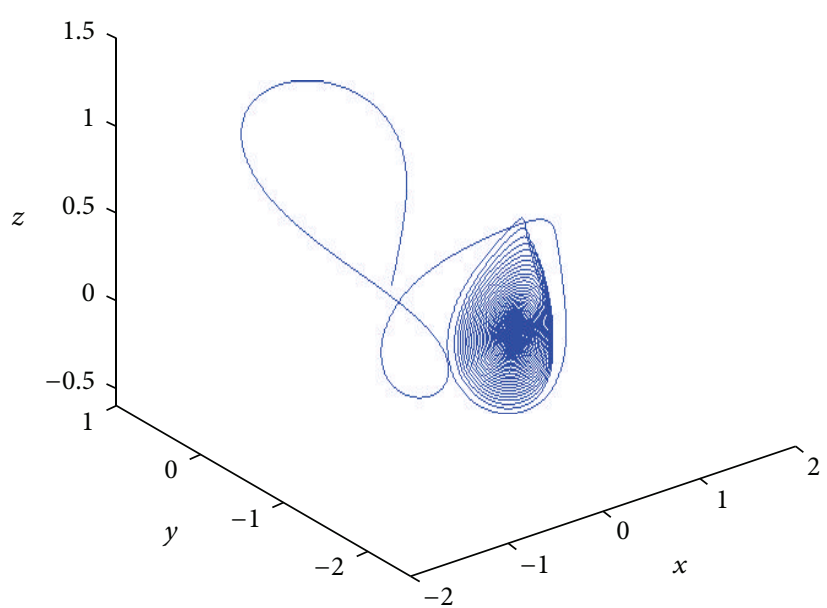

(a)

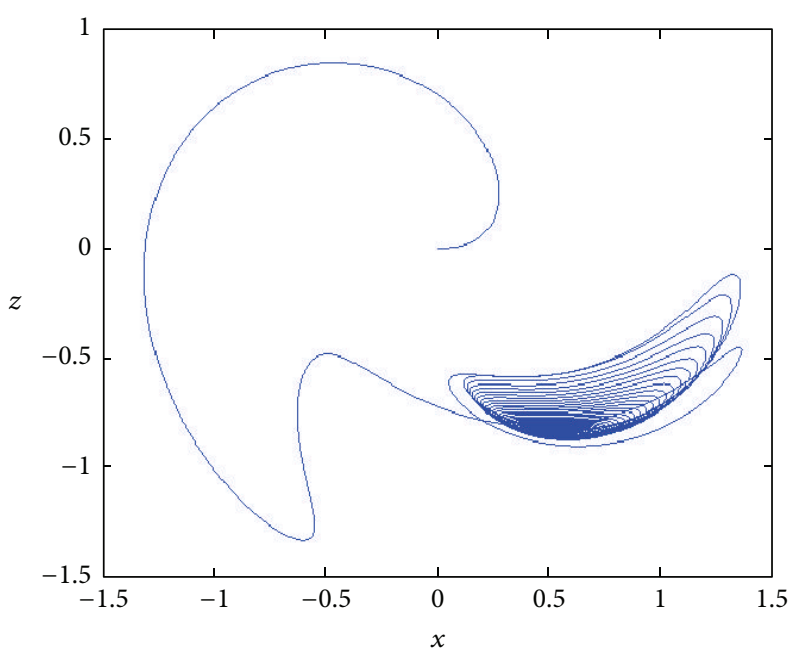

(b)

Figure 6: Phase diagram of system (15) with $a=0.4, b=3$, and $c=-0.8$.

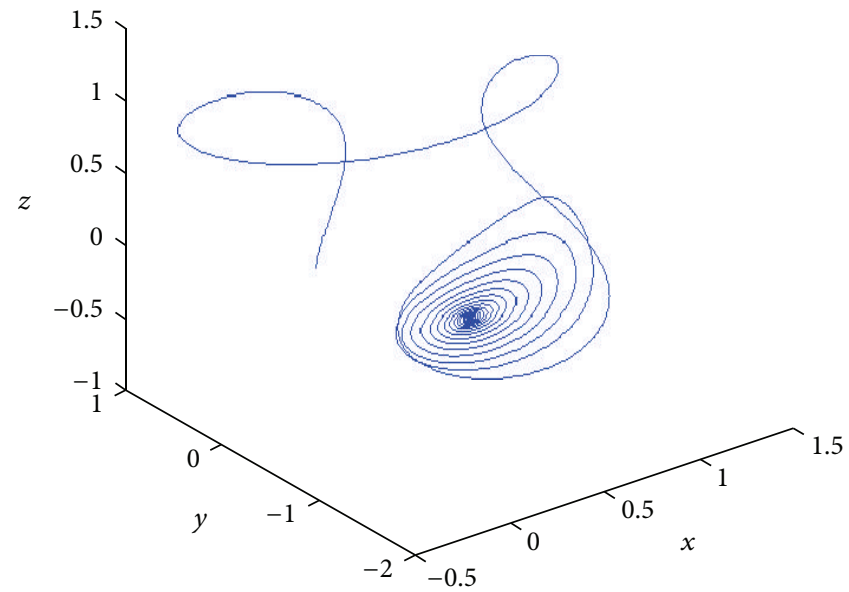

(a)

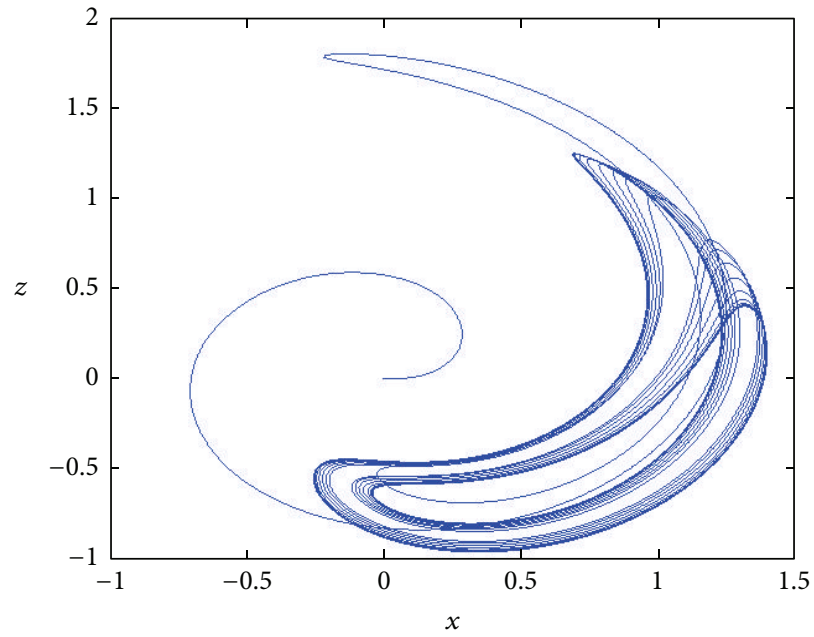

(b)

Figure 7: Phase diagram of system (15) with $a=0.4, b=3$, and $c=-1$.

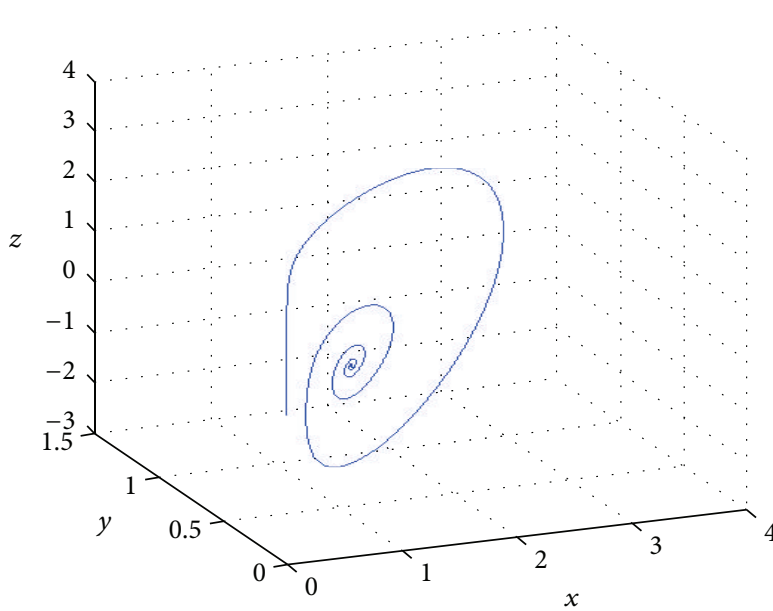

(a)

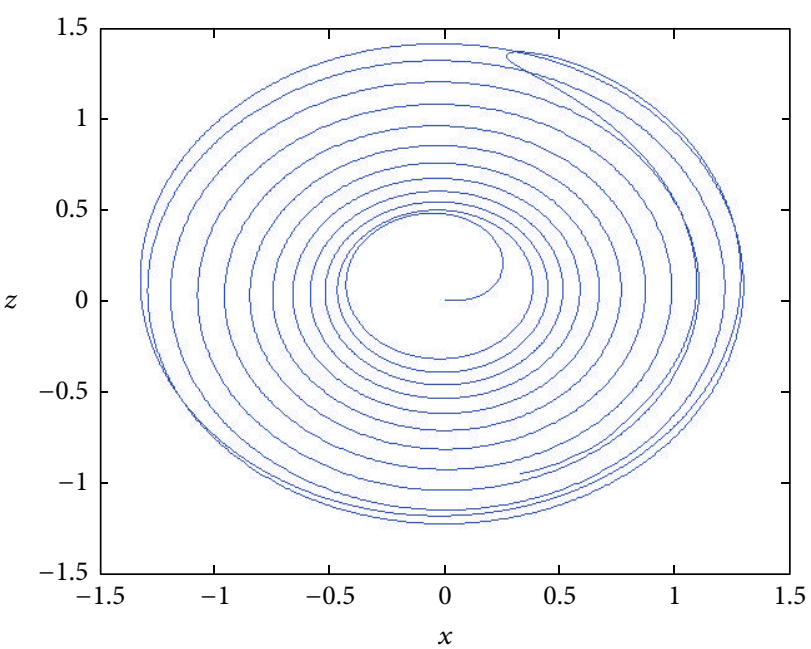

(b)

Figure 8: Phase diagram of system (15) with $a=0.4, b=3$, and $c=-1.9$. 
method, washout-filter method, and frequency domain analysis and approximation method) on the proposed systems and include examples to demonstrate actual solutions of the systems.

\section{Competing Interests}

The authors have declared that no competing interests exist.

\section{Acknowledgments}

The authors gratefully acknowledge the support from the National Natural Science Foundation (no. 11262009, no. 61364001), the Science and Technology Program of Gansu Province (no. 144GKCA018), and the Specialized Research Fund for the Doctoral Program of Higher Education of China (no. 20136204110001).

\section{References}

[1] J. Wang, X. Xiong, and Y. Zhang, "Extending synchronization scheme to chaotic fractional-order Chen systems," Physica A: Statistical Mechanics and its Applications, vol. 370, no. 2, pp. 279-285, 2006.

[2] J. G. Lu and G. Chen, "A note on the fractional-order Chen system," Chaos, Solitons \& Fractals, vol. 27, no. 3, pp. 685-688, 2006.

[3] M. M. Asheghan, M. T. H. Beheshti, and M. S. Tavazoei, "Robust synchronization of perturbed Chen's fractional-order chaotic systems," Communications in Nonlinear Science and Numerical Simulation, vol. 16, no. 2, pp. 1044-1051, 2011.

[4] A. K. Alomari, M. S. M. Noorani, R. Nazar, and C. P. Li, "Homotopy analysis method for solving fractional Lorenz system," Communications in Nonlinear Science and Numerical Simulation, vol. 15, no. 7, pp. 1864-1872, 2010.

[5] W. M. Ahmad, R. El-Khazali, and Y. Al-Assaf, "Stabilization of generalized fractional order chaotic systems using state feedback control," Chaos, Solitons and Fractals, vol. 22, no. 1, pp. 141-150, 2004.

[6] M. P. Aghababa and H. P. Aghababa, "The rich dynamics of fractional-order gyros applying a fractional controller," Proceedings of the Institution of Mechanical Engineers. Part I: Journal of Systems and Control Engineering, vol. 227, no. 7, pp. 588-601, 2013.

[7] M. P. Aghababa, "Fractional modeling and control of a complex nonlinear energy supply-demand system," Complexity, vol. 20, no. 6, pp. 74-86, 2015.

[8] M. P. Aghababa and M. Borjkhani, "Chaotic fractional-order model for muscular blood vessel and its control via fractional control scheme," Complexity, vol. 20, no. 2, pp. 37-46, 2014.

[9] A. E. Matouk, A. A. Elsadany, E. Ahmed, and H. Agiza, "Dynamical behavior of fractional-order Hastings-Powell food chain model and its discretization," Communications in Nonlinear Science and Numerical Simulation, vol. 27, no. 1-3, pp. 153167, 2015.

[10] E. Ahmed and A. S. Elgazzar, "On fractional order differential equations model for nonlocal epidemics," Physica A: Statistical Mechanics and Its Applications, vol. 379, no. 2, pp. 607-614, 2007.

[11] M. S. Tavazoei and M. Haeri, "Limitations of frequency domain approximation for detecting chaos in fractional order systems,"
Nonlinear Analysis: Theory, Methods \& Applications, vol. 69, no. 4, pp. 1299-1320, 2008.

[12] A. S. Hegazi and A. E. Matouk, "Dynamical behaviors and synchronization in the fractional order hyperchaotic Chen system," Applied Mathematics Letters, vol. 24, no. 11, pp. 19381944, 2011.

[13] A. E. Matouk, "Chaos, feedback control and synchronization of a fractional-order modified autonomous Van der Pol-Duffing circuit," Communications in Nonlinear Science and Numerical Simulation, vol. 16, no. 2, pp. 975-986, 2011.

[14] A. S. Hegazi, E. Ahmed, and A. E. Matouk, "On chaos control and synchronization of the commensurate fractional order Liu system," Communications in Nonlinear Science and Numerical Simulation, vol. 18, no. 5, pp. 1193-1202, 2013.

[15] A. E. Matouk and A. A. Elsadany, "Achieving synchronization between the fractional-order hyperchaotic Novel and Chen systems via a new nonlinear control technique," Applied Mathematics Letters, vol. 29, pp. 30-35, 2014.

[16] M. P. Aghababa, "Chaotic behavior in fractional-order horizontal platform systems and its suppression using a fractional finite-time control strategy," Journal of Mechanical Science and Technology, vol. 28, no. 5, pp. 1875-1880, 2014.

[17] Q. Yang, G. Chen, and K. Huang, "Chaotic attractors of the conjugate Lorenz-type system," International Journal of Bifurcation and Chaos in Applied Sciences and Engineering, vol. 17, no. 11, pp. 3929-3949, 2007.

[18] G. Van der Schrier and L. R. M. Maas, “The diffusionless Lorenz equations; Shil'nikov bifurcations and reduction to an explicit map," Physica D: Nonlinear Phenomena, vol. 141, no. 1-2, pp. 1936, 2000.

[19] R. Shaw, "Strange attractors, chaotic behavior, and information flow," Zeitschrift für Naturforschung A, vol. 36, no. 1, pp. 80-112, 1981.

[20] Q. Yang and G. Chen, "A chaotic system with one saddle and two stable node-foci," International Journal of Bifurcation and Chaos, vol. 18, no. 5, pp. 1393-1414, 2008.

[21] T. Katsuyama, "Development of semiconductor laser for optical communication," SEI Technical Review, no. 69, pp. 13-20, 2009.

[22] S. Banerjee, L. Rondoni, and S. Mukhopadhyay, "Synchronization of time delayed semiconductor lasers and its applications in digital cryptography," Optics Communications, vol. 284, no. 19, pp. 4623-4634, 2011.

[23] S. Momani, A. Freihat, and M. AL-Smadi, "Analytical study of fractional-order multiple chaotic FitzHugh-Nagumo neurons model using multistep generalized differential transform method," Abstract and Applied Analysis, vol. 2014, Article ID 276279, 10 pages, 2014.

[24] F. Y. Lin and J. M. Liu, "Nonlinear dynamical characteristics of an optically injected semiconductor laser subject to optoelectronic feedback," Optics Communications, vol. 221, no. 1-3, pp. 173-180, 2003.

[25] I. A. D. H. Al-Saidi and A. H. Kareem, "Instabilities and chaos in optically injected semiconductor lasers," Journal of Basrah Researches (Sciences), vol. 38, no. 2, pp. 20-28, 2012.

[26] T. B. Simpson, J. M. Liu, A. Gavrielides, V. Kovanis, and P. M. Alsing, "Period-doubling cascades and chaos in a semiconductor laser with optical injection," Physical Review A, vol. 51, no. 5, pp. 4181-4185, 1995.

[27] B. Kelleher, C. Bonatto, G. Huyet, and S. P. Hegarty, "Excitability in optically injected semiconductor lasers: contrasting quantum- well- and quantum-dot-based devices," Physical Review E, vol. 83, no. 2, Article ID 026207, 2011. 
[28] B. Kelleher, C. Bonatto, P. Skoda, S. P. Hegarty, and G. Huyet, "Excitation regeneration in delay-coupled oscillators," Physical Review E, vol. 81, no. 3, Article ID 036204, 2010.

[29] S. Eriksson and Å. M. Lindberg, "Observations on the dynamics of semiconductor lasers subjected to external optical injection," Journal of Optics B: Quantum and Semiclassical Optics, vol. 4, no. 2, pp. 149-154, 2002.

[30] X. Li, W. Pan, B. Luo, D. Ma, Y. Wang, and N. Li, "Nonlinear dynamic behaviors of an optically injected vertical-cavity surface-emitting laser," Chaos, Solitons \& Fractals, vol. 27, no. 5, pp. 1387-1394, 2006.

[31] S. Wieczorek, B. Krauskopf, and D. Lenstra, "Unifying view of bifurcations in a semiconductor laser subject to optical injection," Optics Communications, vol. 172, no. 1, pp. 279-295, 1999.

[32] K. E. Chlouverakis and M. J. Adams, "Stability maps of injection-locked laser diodes using the largest Lyapunov exponent," Optics Communications, vol. 216, no. 4-6, pp. 405-412, 2003.

[33] Y.-D. Chu, X.-F. Li, J.-G. Zhang, and Y.-X. Chang, "Nonlinear dynamics analysis of a new autonomous chaotic system," Journal of Zhejiang University SCIENCE A, vol. 8, no. 9, pp. 14081413, 2007.

[34] M. Caputo, "Linear models of dissipation whose Q is almost frequency independent-II," Geophysical Journal International, vol. 13 , no. 5, pp. 529-539, 1967.

[35] F. Ben Adda, "Geometric interpretation of the fractional derivative," Journal of Fractional Calculus, vol. 11, pp. 21-51, 1997.

[36] I. Podlubny, "Geometric and physical interpretation of fractional integration and fractional differentiation," 2001, https://arxiv.org/abs/math/0110241.

[37] D. Matignon, "Stability results for fractional differential equations with applications to control processing," in Proceedings of the Symposium on Modelling, Analysis and Simulation: (CESA '96) IMACS Multiconference, Computational Engineering in Systems Applications, vol. 2, Lille, France, July 1996.

[38] A. E. Matouk, "Stability conditions, hyperchaos and control in a novel fractional order hyperchaotic system," Physics Letters A, vol. 373, no. 25, pp. 2166-2173, 2009.

[39] E. Matouk A, "Dynamical behaviors, linear feedback control and synchronization of the fractional order Liu system," Journal of Nonlinear Systems and Applications, vol. 3, pp. 135-140, 2010.

[40] A. S. Hegazi, E. Ahmed, and A. E. Matouk, "The effect of fractional order on synchronization of two fractional order chaotic and hyperchaotic systems," Journal of Fractional Calculus and Applications, vol. 1, no. 3, pp. 1-15, 2011.

[41] A. E. Matouk, "Chaos, feedback control and synchronization of a fractional-order modified autonomous Van der Pol-Duffing circuit," Communications in Nonlinear Science and Numerical Simulation, vol. 16, no. 2, pp. 975-986, 2011.

[42] S. Elaydi, An Introduction to Difference Equations, Springer Science \& Business Media, 2005.

[43] G. Wen, "Criterion to identify Hopf bifurcations in maps of arbitrary dimension," Physical Review E, vol. 72, no. 2, Article ID 026201, 2005. 


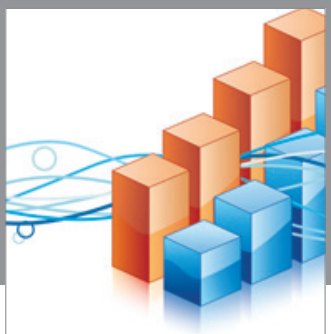

Advances in

Operations Research

vatem alat4

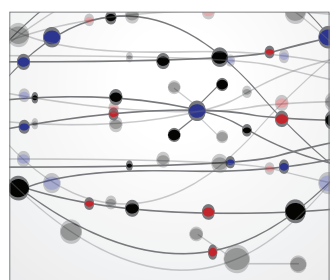

\section{The Scientific} World Journal
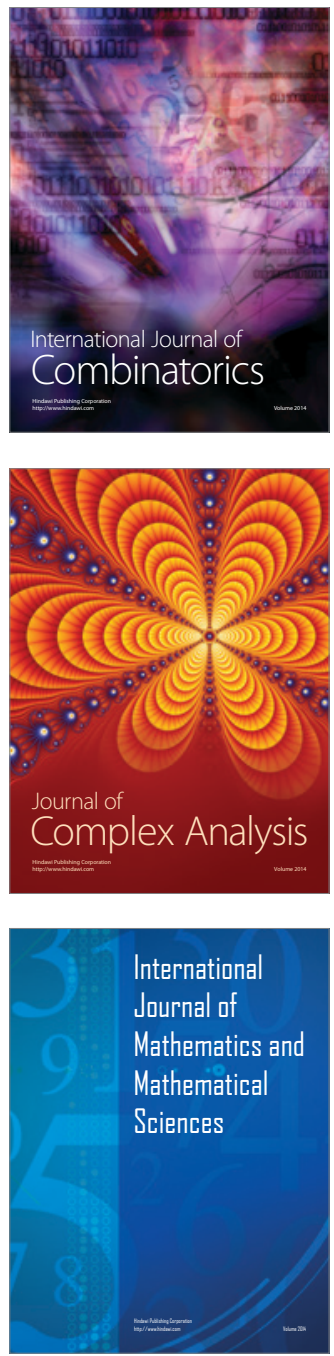
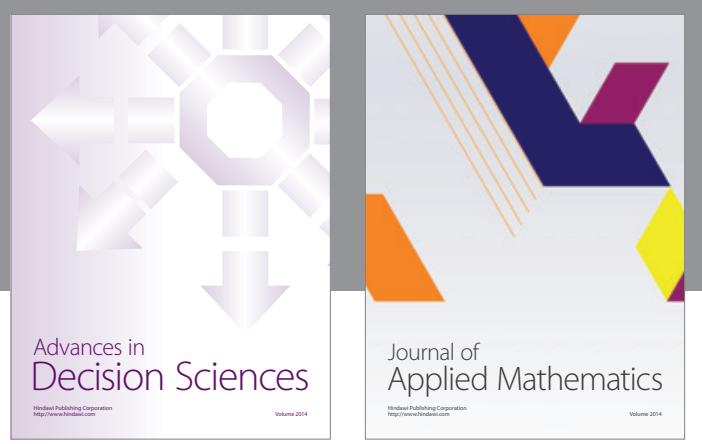

Algebra

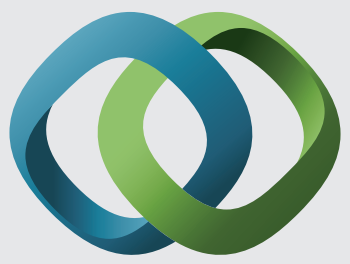

\section{Hindawi}

Submit your manuscripts at

http://www.hindawi.com
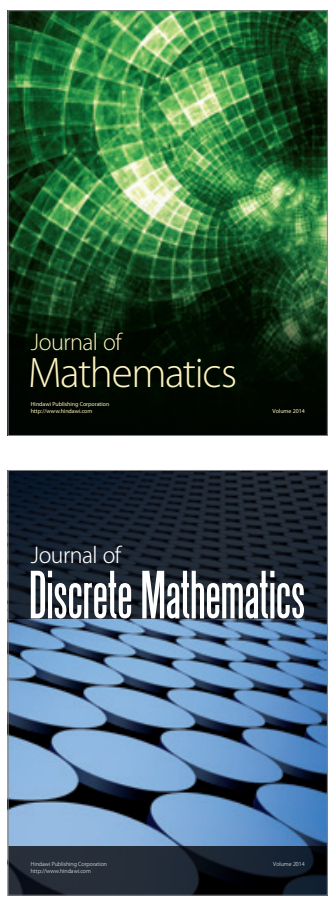

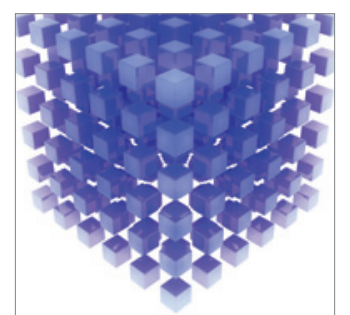

Mathematical Problems in Engineering
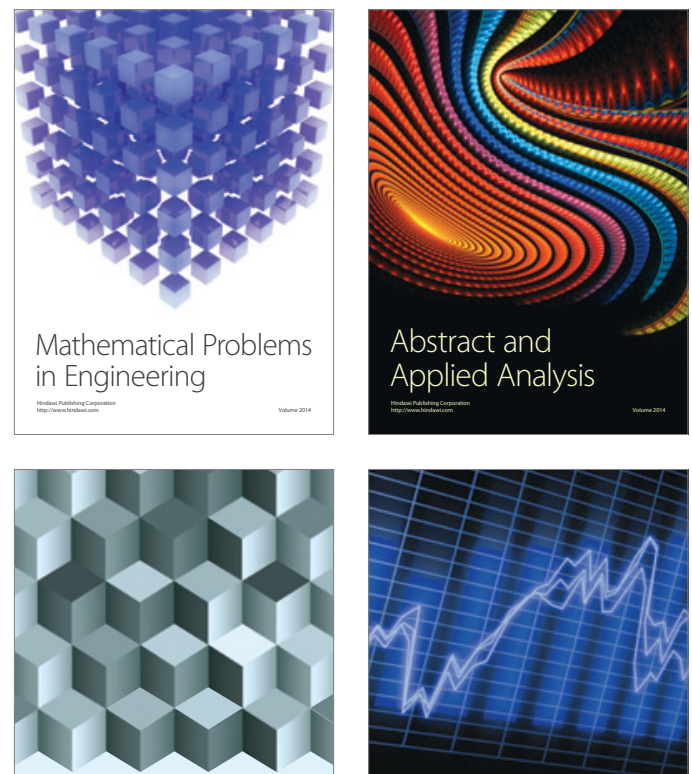

Journal of

Function Spaces

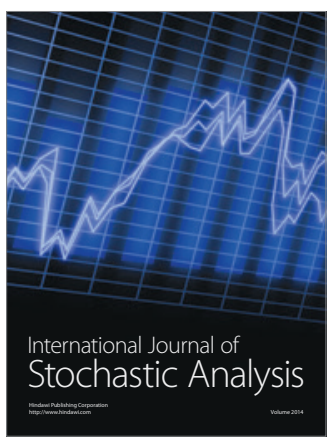

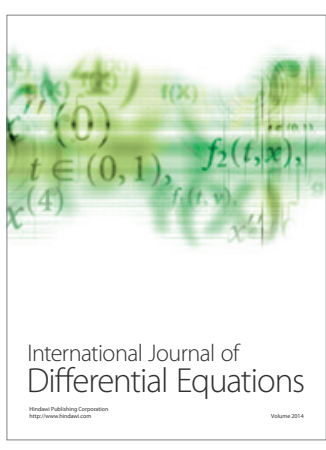
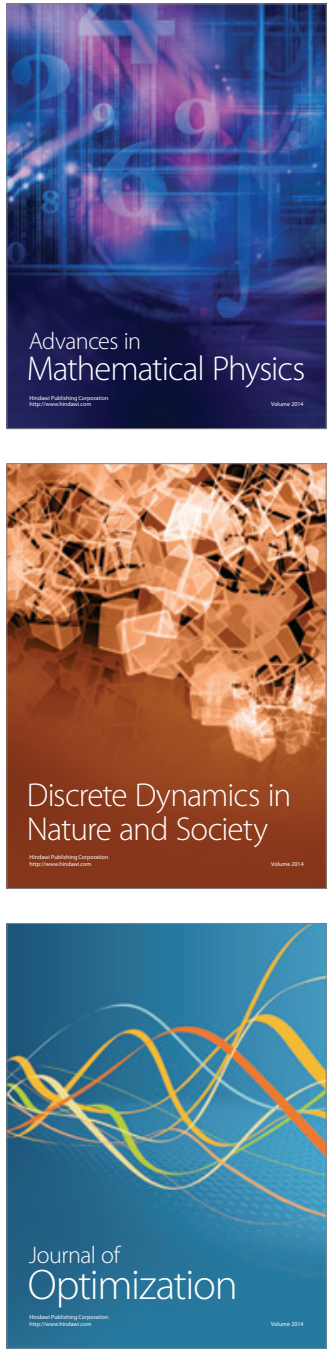